\title{
Life Cycle Assessment of Different Nitrogen-Doped Reduced Graphene Oxide Production Routes within Early Research
}

\author{
Xiaohua $\mathrm{Ge}^{1,2}$, Junyao $\mathrm{Lu}^{3}$, Lin $\mathrm{Ma}^{4}$, Jianchao Ma ${ }^{5 *}$ \\ ${ }^{1}$ Taiyuan Institute of Technology No.31, Xinlan Road, Jiancaoping District, Taiyuan, Shanxi, China \\ ${ }^{2}$ The Research Center for Eco-Environment Science in Shanxi Province, \\ No.24, Sanqiang ST, Xinghualing District, Taiyuan, Shanxi, China \\ ${ }^{3}$ Business College of Shanxi University, Junyao Lu, 16 km, Taiyu Road, Xiaodian District, Taiyuan, Shanxi, China \\ ${ }^{4}$ The Research Center for Eco-Environment Science in Shanxi Province, \\ No.24, Sanqiang ST, Xinghualing District, Taiyuan, Shanxi, China \\ ${ }^{5}$ Taiyuan University of Technology, No.79 West Yingze ST, Wanbailing District, Taiyuan, Shanxi, China
}

Received: 18 May 2020

Accepted: 20 July 2020

\begin{abstract}
Nitrogen-doped Reduced Graphene Oxide (N-rGO) has raised tremendous interest in water treatment owing to its fascinating properties. There are two main ways to synthesis N-rGO, which is hydrothermal method (HM) and annealing method (AM) in water treatment field. But as an efficient catalyst in water treatment, the process of $\mathrm{N}-\mathrm{rGO}$ production also has some environmental impacts. This study assesses the life cycle environmental impacts of N-rGO produced by HM and AM, and explore the implications of varying the key production parameters with a thorough sensitivity analysis. The results show that the environmental impacts of HM is higher than that of AM, so AM route is more available to scale-up production than HM route. For AM route, electricity reduction is the most efficient way to decrease the environmental impacts, and the next is ethanol recovery. For HM route, ethanol recovery is the most efficient way to decrease the environmental impacts, and the next is electricity reduction.
\end{abstract}

Keywords: life cycle assessment, Nitrogen-doped reduced graphene oxide, hydrothermal method, annealing method

\section{Introduction}

Recent years, graphene and graphene-like materials have attracted worldwide attention, owing to its fascinating properties [1], such as high surface area,

*e-mail: majianchao@tyut.edu.cn high thermal conductivity, fast charged carrier mobility and strong Young's modulus, which have been well documented [2]. All these aspects make graphene material promising for various applications, including energy conversion and storage, electrocatalysis, sensors and electronics [3].

In order to improve the property of graphene, Nitrogen atoms, as heteroatoms, have been introduced 
into the carbon lattice of graphene. The introduction of $\mathrm{N}$ dopants into the well-defined graphitic carbon framework can effectively disorientate the homogeneously conjugated electron network and modulate the surface properties by tweaking the charge distribution and spinning culture of the doped domains [4]. Many studies show that N-doping has been demonstrated to be able to endow pristine graphene with impressively enhanced properties and greatly broadens its applications [5]. Recently as one of the emerging applications of $\mathrm{N}$-doping graphene in water treatment has raised tremendous interest [6], which can be employed as the most effective activators for peroxymonosulfate (PMS) and peroxydisulfate (PDS) to generate sulfate radical $\left(\mathrm{SO}_{4} \bullet-\right)$, hydroxyl radical $(\bullet \mathrm{OH})$, and/or superoxide radical $\left(\mathrm{O}_{2}{ }^{--}\right)$to decompose of a wide range of contaminants without secondary contamination [7-10].

There are many different synthesis methods for $\mathrm{N}$-doping have been investigated, such as chemical vapor deposition (CVD) [11], segregation growth [12], solvothermal [13], arc discharge [14], thermal treatment [15], plasma treatment [16] and $\mathrm{N}_{2} \mathrm{H}_{4}$ treatment [17]. In water treatment field, thermal treatment is one of the most common way to synthesize Nitrogen-doped Reduced Graphene Oxide(N-rGO). Based on thermal treatment, a variety of $\mathrm{N}-\mathrm{rGO}$ production routes in laboratory scale have been investigated in literature [6]. But the general methods can be grouped into two methods: (1) Hydrothermal method (HM), with GO and hydrothermal treatment, (2) Annealing method (AM), with GO and annealing treatment.

$\mathrm{N}$-rGO is an efficient catalyst in water treatment, but the process of N-rGO production also has some environmental impacts. Tischner and Deutschland have reported that about $80 \%$ of all environmental effects associated with a product are determined in the design phase of development [18], so it is important to improve the understanding of their environment impacts to identify which of those routes and nitrogen precursors is of relevance for industrial-scale production of $\mathrm{N}$-rGO. Life cycle assessment (LCA) is a potential tool for the assessment of the environment impacts of technology, which has already been successful applied in many fields [19]. Application of LCA to nanotechnology can contributes to the development of "green nanotechnology" [20], and there have been various previous studies on nanocarbons production before. But to the best of our knowledge, the life cycle environmental impacts of N-rGO production have not been assessed before, although the GO production is partly similar to the graphene production assessed by Arvidsson and Cossutta [21-23].

In this study, based on our previous studies in water treatment, we assess the life cycle environmental impacts of N-rGO produced by $\mathrm{HM}$ and $\mathrm{AM}$, and explore the implications of varying the key production parameters with a thorough sensitivity analysis. The results of this study will help to identify the sources of significant potential environmental impacts and developing the green $\mathrm{N}$-rGO production route.

\section{Materials and Methods}

\section{Goal and Scope}

There are mainly two goals in this study. The first is to assess the environmental impacts for different $\mathrm{N}-\mathrm{rGO}$ production methods. The second is to understand how the environmental impact changes along the process inputs and identify possible improvement potentials for N-rGO production.

As shown in Fig. 1, the scope of the study is to assess cradle-to-gate life cycle impacts of N-rGO production, which include the activities from raw material extraction and processing to N-rGO production. The transportation of materials (such as chemicals) was not included in HM and AM, because the N-rGO processes are still at the laboratorial stage and the locations of the production units have not yet been determined. Specific applications are also not considered, so the use phase and end-of-life activities are excluded from the analysis. The functional unit of the study is $1 \mathrm{~kg} \mathrm{~N}$-rGO.

\section{Data and Sensitivity}

There are two main sources of data. Our laboratory experiments provide the process data as shown in Table 1, the Ecoinvent database (version 3.5) provided the inventory data for all input materials and energy. Note that the units in our previous studies are $\mathrm{g}$ and $\mathrm{ml}$, but these units are scaled up linearly to $\mathrm{kg}$ in the study. Since N-rGO is a novel product, the technology system is under development. LCAs at this stage pose a multitude of challenges due to date unavailability, scale issues and technology uncertainties [24]. Hence, sensitivity analysis will be employed to investigate the inherent uncertainty in the technical system brought on by its emerging nature.

\section{GO Production}

GO was prepared by a modified Hummers method [25], which is the basic material to synthesize the N-rGO in $\mathrm{HM}$ and AM. In Hummers method, graphite is synthesized using graphite, permanganate and sulfuric acid, some deionized water is used to prevent violent effervescence and wash $\mathrm{GO}$, some hydrogen peroxide $(30 \%)$ is used to reduce the residual permanganate and $\mathrm{MnO}_{2}$ to soluble $\mathrm{MnSO}_{4}$. Electricity is used to stirring, heating, centrifugalizing and drying.

Data on quantity inputs for GO production were measured using general lab equipment like beakers and scales. Electricity consumption for hot plates and centrifuge is calculated by power factor and time, Electricity consumption for drying is assume that 


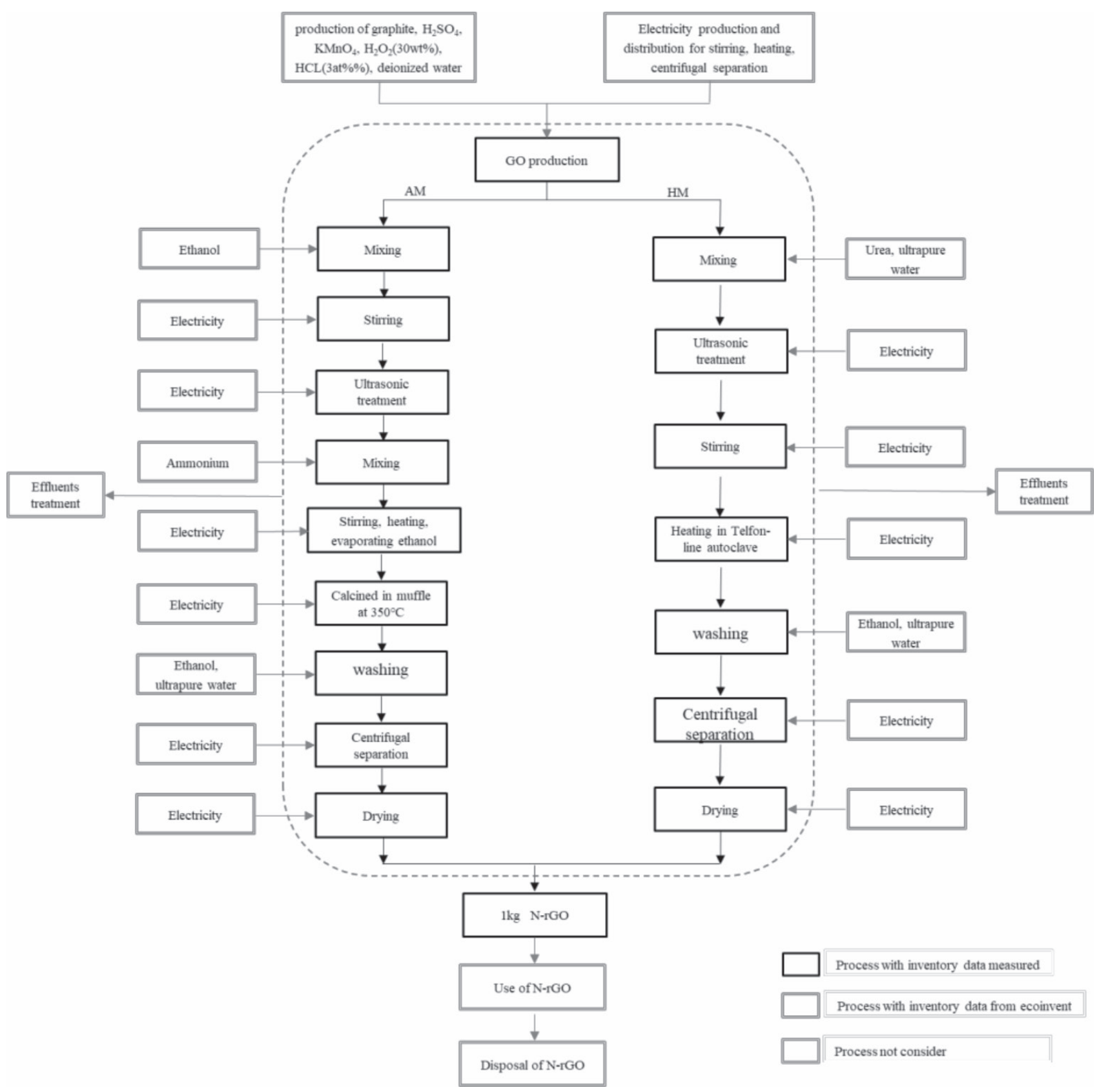

Fig. 1. Flowchart describing the cradle-to-gate life cycle of N-rGO produced by Hydrothermal method (HM) and Annealing method (AM).

moisture content of GO was $100 \%$ and the energy for drying $1 \mathrm{~kg}$ water is $8 \mathrm{~kJ}$.

Sensitivity analysis can help to understand the main contributing processes for environmental impacts in scale-up production can reduce [22, 23]. Sensitivity analysis for GO production is mainly considered the solvent recovery and the reduction of electricity use. Solvent recovery in industry can range from $0 \%$ to $90 \%$ [39]. In this study, we consider the recovery of water used for washing and surplus sulfuric acid for oxidation is from $0 \%$ to $90 \%$, the reduction of electricity is from $0 \%$ to $90 \%$. The $0 \%$ is set in the baseline case, the $90 \%$ recovery and reduction are assessed in the sensitivity analysis.

\section{N-rGO Production from Hydrothermal Method}

In hydrothermal method [9], GO and urea are mixed and transferred into a Teflon-lined autoclave and heated at $180 \mathrm{C}$ for $18 \mathrm{~h}$ to synthesized the N-rGO, ultrapure water is used to mix GO and urea and wash N-rGO, ethanol is used to wash N-rGO. Electricity is used in hot plates, centrifuge, Teflon-lined autoclave, ultrasound and oven to stir, heat, centrifugalize and dry. Kang et al. also describes another version of N-rGO from hydrothermal method [10]. In this case, it uses 1.5 folds urea. We label the two processes HM1 (original method and baseline case) and HM2 (1.5 folds). 
Table 1 . The synthesis inventory date of $1 \mathrm{~kg}$ N-rGO produced by Hydrothermal method and Annealing method.

\begin{tabular}{|c|c|c|c|}
\hline \multicolumn{2}{|c|}{ Graphite oxide production } & Unit & Amount \\
\hline \multirow[t]{6}{*}{ Input } & Graphite & $\mathrm{Kg}$ & 0.50 \\
\hline & $\mathrm{H}_{2} \mathrm{SO}_{4}(98 \%)$ & $\mathrm{Kg}$ & 12.50 \\
\hline & $\mathrm{Kmno}_{4}$ & $\mathrm{Kg}$ & 1.58 \\
\hline & Deionized water & $\mathrm{Kg}$ & 149.03 \\
\hline & Hydrogen peroxide (50 \%) & $\mathrm{Kg}$ & 1.17 \\
\hline & Electricity & MJ & 97.05 \\
\hline Output & Graphite oxide (GO) & $\mathrm{Kg}$ & 1.00 \\
\hline \multicolumn{4}{|c|}{ N-rGO production by Hydrothermal method } \\
\hline \multirow[t]{5}{*}{ Input } & GO & $\mathrm{Kg}$ & 1.25 \\
\hline & Urea & $\mathrm{Kg}$ & 1.25 \\
\hline & Ultrapure water & $\mathrm{Kg}$ & 212.50 \\
\hline & Ethanol & $\mathrm{Kg}$ & 102.00 \\
\hline & Electricity & MJ & 2265.49 \\
\hline Output & N-rgo & $\mathrm{Kg}$ & 1.00 \\
\hline \multicolumn{4}{|c|}{ N-rGO production by Annealing method } \\
\hline \multirow[t]{5}{*}{ Input } & GO & $\mathrm{Kg}$ & 2.50 \\
\hline & Ammonium nitrate & $\mathrm{Kg}$ & 2.50 \\
\hline & Ultrapure water & $\mathrm{Kg}$ & 125.00 \\
\hline & Ethanol & $\mathrm{Kg}$ & 204.00 \\
\hline & Electricity & $\mathrm{MJ}$ & 259.97 \\
\hline Output & N-rgo & $\mathrm{Kg}$ & 1.00 \\
\hline
\end{tabular}

Data on quantity inputs for N-rGO production were measured using general lab equipment like beakers and scales. Electricity consumption for hot plates, centrifuge and ultrasound is calculated by power factor and time, Electricity consumption for drying is assumed that moisture content of N-rGO was $50 \%$ and the energy for drying $1 \mathrm{~kg}$ water is $8 \mathrm{~kJ}$. Electricity consumption for Teflon-lined autoclave is calculated using

$$
\mathrm{E}=(\mathrm{H} 1+\mathrm{H} 2) / \mathrm{f} ; \quad \mathrm{H}_{1}=\mathrm{m} \times \mathrm{c}_{\mathrm{p}} \times \Delta \mathrm{T} ;
$$

...where $\mathrm{E}$ is the energy electricity consumption, MJ. $\mathrm{H} 1$ is the heat to heating the material in Teflon-lined autoclave, MJ. H2 is heat losses during the operation which is calculated using the mechanical insulation design guide provided by the National Institute of Building Service, MJ [22]. F is heat conversion efficiency, which is assumed $70 \% . \mathrm{M}$ is the mass of the heated materials, $\mathrm{kg}, \mathrm{c}_{\mathrm{p}}$ is the specific heat capacity of the materials, $\mathrm{MJ} /(\mathrm{kg} \cdot \mathrm{K}), \Delta \mathrm{T}$ is the change in temperature that the materials experience, $\mathrm{K}$.
Sensitivity analysis for N-rGO production is mainly consider the solvent recovery and the reduction of electricity use. In this study, we consider the recovery of water used for mixing and washing, and ethanol for washing, the recovery of these solvent is from $0 \%$ to $90 \%$. The reduction of electricity is from $0 \%$ to $90 \%$. The $0 \%$ is set in the baseline case, the $90 \%$ recovery and reduction are assessed in the sensitivity analysis.

\section{N-rGO Production from Annealing Method}

In annealing method [14], GO and ammonium nitrate are mixed in ethanol, after stir and sonicate for some time, ethanol is evaporated on a hotplate, and then the dried mixture was finely grinded and transferred into a muffle furnace and calcined at $350^{\circ} \mathrm{C}$ for $1 \mathrm{~h}$. Ethanol and ultrapure water are used to wash N-rGO. Electricity is used in hot plates, centrifuge, muffle furnace, ultrasound and oven to stir, heat, anneal centrifugalize and dry.

Data on quantity inputs for N-rGO production were measured using general lab equipment like beakers and scales. Electricity consumption for hot plates, centrifuge and ultrasound is calculated by power factor and time, Electricity consumption for drying is assume that moisture content of $\mathrm{N}-\mathrm{rGO}$ was $50 \%$ and the energy for drying $1 \mathrm{~kg}$ water is $8 \mathrm{~kJ}$. Electricity consumption for muffle furnace is calculated like Teflon-lined autoclave.

Sensitivity analysis for N-rGO production is mainly considered the solvent recovery and the reduction of electricity use. In this study, we consider the recovery of ethanol used for mixing and washing, and water for washing, the recovery of these solvents is from $0 \%$ to $90 \%$. The reduction of electricity is from $0 \%$ to $90 \%$. The $0 \%$ is set in the baseline case, the $90 \%$ recovery and reduction are assessed in the sensitivity analysis.

\section{Impact Categories}

The environmental impacts analyzed include: climate change, cumulative energy demand(CED), water use, human toxicity and ecotoxicity. Climate change is one of the word serious environmental issues which is a very common and important impactor in LCA, and also for N-rGO production. Cumulative energy demand is also one of the key indicators in LCA, it is considered in this study because N-rGO production uses a lot of electricity and it is energy intensive. Water use includes the water used during production in foreground and background [23]. Human toxicity and ecotoxicity consider a lot of chemicals used in N-rGO production which is toxic to humans and the environment.

Climate change and water use are investigated based on impact assessment from the ReCiPe midpoint method, The results are expressed in $\mathrm{CO}_{2}$-equivalents, Water depletion measures the volume of water (by cubic meters) used while considering water categories that contribute to water shortages [23]. The cumulative energy demand (CED) method was used for calculating 
cumulative energy demand, measured in MJ [23]. The human toxicity and ecotoxicity indicator are from the USEtox model, these impact categories are measure in comparative toxic units (CTU), with a subscript " $h$ " and "e" to indicate human or ecotoxicity, respectively [22].

\section{Results and Discussion}

\section{Climate Change}

As shown in Fig. 2, the climate change in AM and $\mathrm{HM}$ are 351.1 and $591.0 \mathrm{~kg} \mathrm{CO}-\mathrm{Eq}$, the $\mathrm{HM}$ has a higher impact than that of the AM, this is mainly because the HM route need 23 times more electricity than AM. Therefore, the main contributor in HM is electricity use (approximately 75.6\%), due to heating, stirring, ultrasound, centrifuge and drying, the biggest contributor among them is the heating for Teflonlined autoclave, which is approximately 93.7\%. Ethanol production and GO process contribute as well, approximately $18.3 \%$ and $5.2 \%$. The production of ultrapure water and urea is negligible. For the AM, Ethanol production is main contributor (approximately $61.6 \%$ ), the next is GO process, electricity production,
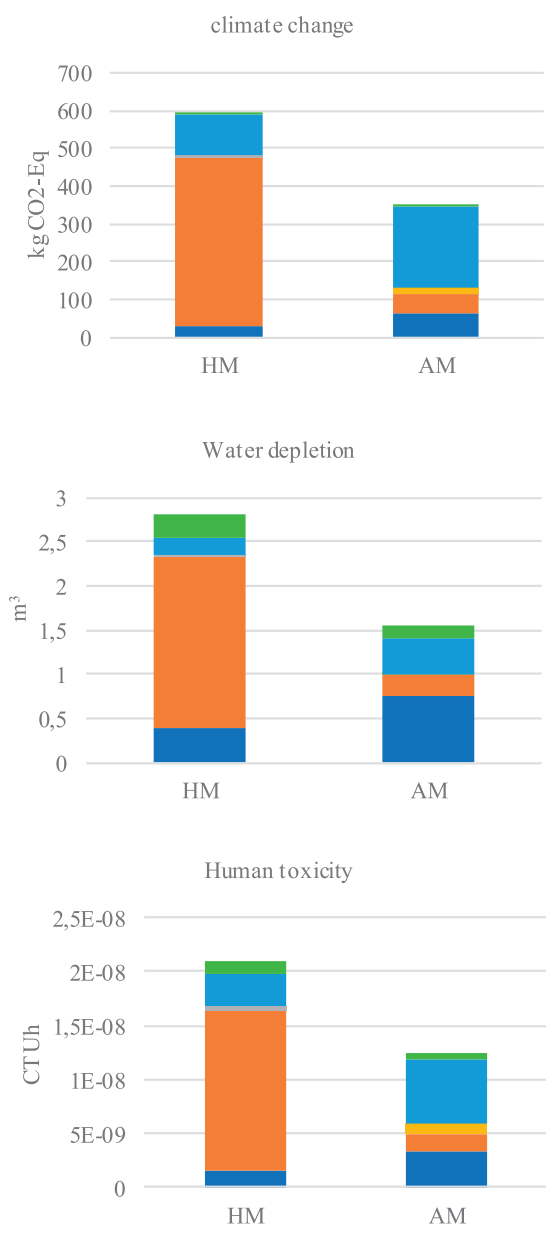

ammonium nitrate production and ultrapure water production which are in the range of $17.8 \%, 15.0 \%$, $4.1 \%, 1.5 \%$, respectively. Thus, the reduction of electricity for two methods is the main way to decrease the climate change impact, which will be assessed in sensitivity analysis.

\section{Cumulative Energy Demand}

For CED, the impacts of AM and HM are 11623.0 and $13085.3 \mathrm{MJ}-\mathrm{Eq}$, the HM is slightly higher than AM (Fig. 2). This is because the contributor of ethanol is greatly increased in two methods and the ethanol use in AM is double that in HM. According to the ethanol background data in the Ecoinvent database (version 3.5), the production of ethanol are energy intensive, a lot of fossil energy are used as raw materials and fuels. Thus, although electricity use in HM is the main contributor (approximately 58.1\%), compared to the impact of climate change, the contributor of ethanol production is significant increased from $17.7 \%$ to $35.5 \%$. The contributor of other three materials is less than $6.5 \%$. For AM, the biggest contributor is the ethanol production, which is approximately $79.9 \%$. GO process and electricity production are next, approximately
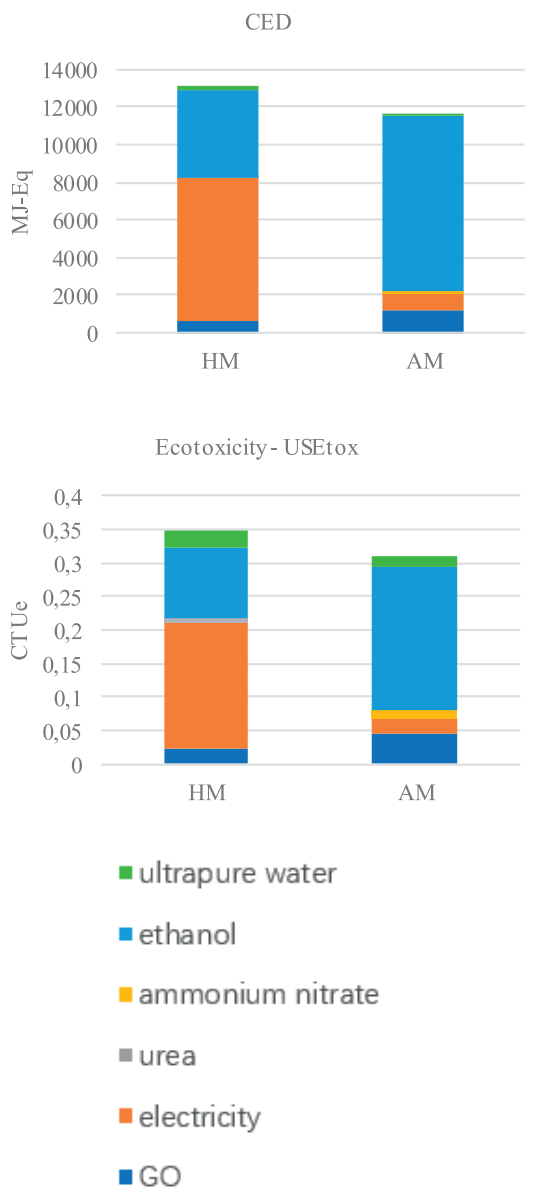

Fig. 2. Life cycle assessment results for the baseline scenarios of the two studied routes for N-rGO CED is cumulative energy demand. HM is Hydrothermal method. AM is Annealing method. 
$10.54 \%$ and $7.5 \%$. The production of ultrapure water and ammonium nitrate is approximately $2 \%$. Therefore, the reduction of ethanol and electricity use can decrease the CED in both methods, which will be assessed in sensitivity analysis.

\section{Water Depletion}

Regarding the water depletion, As shown in Fig. 2, the impacts of AM and HM are 1.6 and $2.3 \mathrm{~m}^{3}$ respectively, the HM is significantly higher than that of AM and we also find the contributors of $\mathrm{GO}$ and ultrapure water use are increased in two methods, this is because the impact of water depletion mainly relates to the water use in different processes. Similarly the climate change and CED, the most contributor for water depletion in $\mathrm{HM}$ is electricity production (approximately 69.7\%), but the next is GO process, ultrapure water production and ethanol production, which are $13.5 \%, 9.3 \%, 7.3 \%$, respectively. Urea production is slight. In AM, GO process mainly contributes to $48.8 \%$, because more $\mathrm{GO}$ is used in AM. The next is ethanol production, electricity production and ultrapure water production, which are $26.4 \%, 14.4 \%$ and $9.9 \%$, ammonium nitrate production is slight. Although the GO use can not be reduced in both methods, water use in GO can be reduced. Thus, we will test the contributor of reduction of water use, electricity use and ethanol use for water depletion impact in sensitivity analysis.

\section{Ecotoxicity}

The results for the ecotoxicity impact are similar for two production methods, which are 0.35 and 0.31 CUTe for $\mathrm{HM}$ and AM respectively. As shown in Fig. 2, in $\mathrm{HM}$, the main contributors are electricity (approximately $69.7 \%$ ) and ethanol (17.2\%) production, where phenol emission to water contributes $41.2 \%$. The impacts of the other three materials production are approximately $15.6 \%$. For AM, ethanol and GO production are the main contributor, accounting for $69.2 \%$ and $14.93 \%$ respectively, this is again mainly due to phenol emission to water, which contributes about $52.7 \%$. the impacts of the other three materials production are approximately $15.9 \%$. Therefore, the reduction of ethanol and electricity can reduce the ecotoxicity impact, which are tested in sensitivity analysis.

\section{Human Toxicity}

For human toxicity, the impact in HM is larger than that in AM, accounting for 2.1E-08 and 1.2E-08 respectively. As shown in Fig. 2, the main contributor for HM is electricity production, which contributes $70.8 \%$, the formaldehyde emission to air is the main contributor for electricity in HM. The next is ethanol production (approximately 14.5\%), the contributors of the other three materials production are less than $15 \%$.
For AM, ethanol production is the largest contributor, accounting for $48.5 \%$, the formaldehyde emission to air in heat production is the main contributor for ethanol production in AM. The next are GO and electricity production, which contribute $26.1 \%$ and $13.6 \%$ respectively. Thus, the use of electricity and ethanol is key factor to human toxicity impact, which will be tested in sensitivity analysis.

\section{Sensitivity Analysis}

Sensitivity analysis is an important guidance for producers [39]. For N-rGO, it is important to understand which parameters such as different chemicals and energy uses have a large impact on the results in the early stage studies, so we can pay more attention to those significant impacts in further scale-up studies. In this study, the parameters investigated in the sensitivity analysis for GO production are the recovery of water used for washing, surplus sulfuric acid for oxidation $(0-90 \%)$ and the reduction of electricity $(0-90 \%)$. For $\mathrm{HM}$, we consider the recovery of water used for mixing and washing (0-90\%), ethanol for washing (0-90\%), and the reduction of electricity (0-90\%). For AM, we consider the recovery of ethanol used for mixing and washing (0-90\%), and water for washing (0-90\%), and the reduction of electricity $(0-90 \%)$. The results of sensitivity analysis are showed in Figs 5 and 6.

GO is the basic material for N-rGO production, we mainly considered the use of water, sulfuric acid and electricity as sensitivity analysis parameters. For $\mathrm{HM}$, the influence of GO is relatively small, the bigger impact for water recovery in GO is water depletion, which reduced $5.9 \%$ for baseline scenario, the other impacts are less than $0.2 \%$. the impacts of electricity reduction in GO is between $2.6-3.6 \%$ for baseline scenario, the biggest impact is climate change. Sulfuric acid recovery in GO has lager impacts on ecotoxicity and human toxicity, which is approximately $2.1 \%$ and $2.2 \%$ for baseline scenario, the others are less than $1 \%$. In all, most of those impacts are relatively small to other scenarios. By contrast, improving the efficient of water recovery in GO is good way to reduce the impact of water depletion.

For AM, the influence of GO is relatively significant, this is because it needs more GO in HM than that in AM. Water recovery in GO mainly reduces the water depletion (approximately $21.3 \%$ for baseline scenario), which is the largest reduction for water depletion in all sensitive analysis scenarios, the other impacts are less than $0.4 \%$. The impacts electricity reduction in GO are from $5.9 \%$ to $12.6 \%$ for the baseline scenario, the biggest is the climate change. Sulfuric acid recovery mainly reduces the impacts of ecotoxicity and human toxicity, which are respectively $4.8 \%$ and $7.6 \%$ for the baseline scenario. Thus, improving the efficient of electricity reduction in GO can efficiently decrease all impacts, improving the efficient of water recovery in GO can significantly decrease the impact of water 


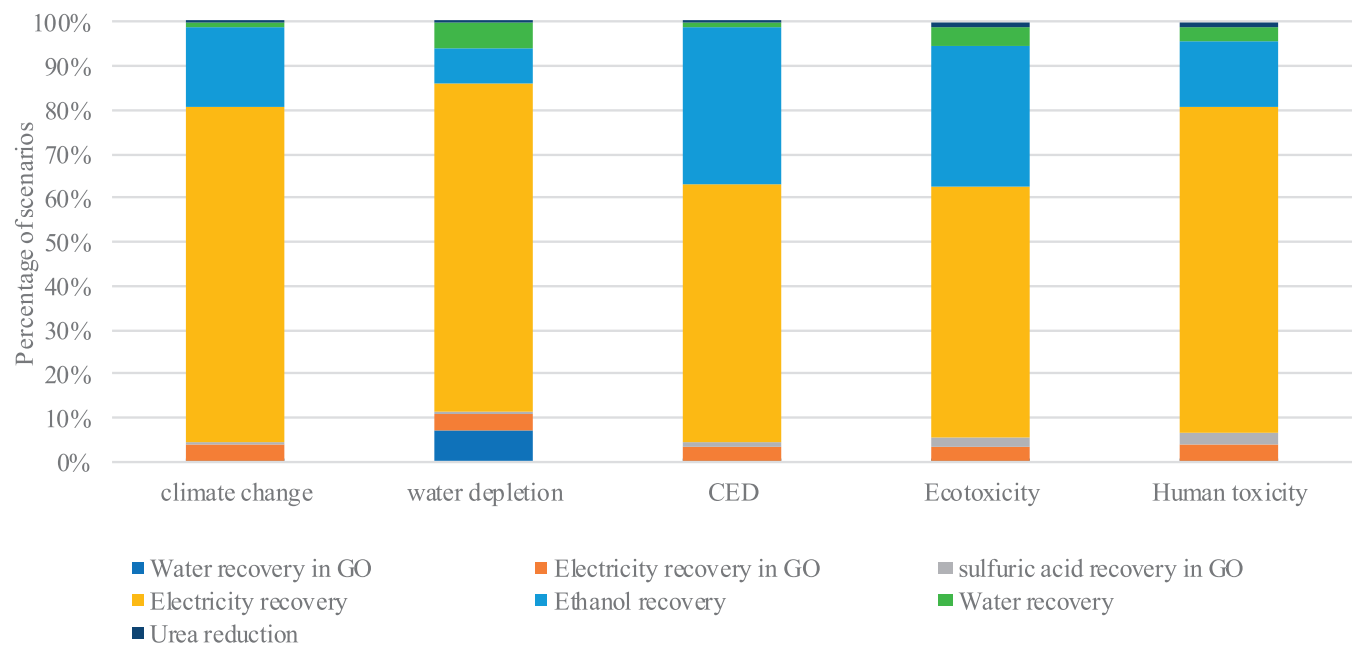

Fig. 3. The results of the sensitivity analysis for N-rGO synthesized by HM, showing the importance of the single scenarios for the reduction of each impact category in percentage.

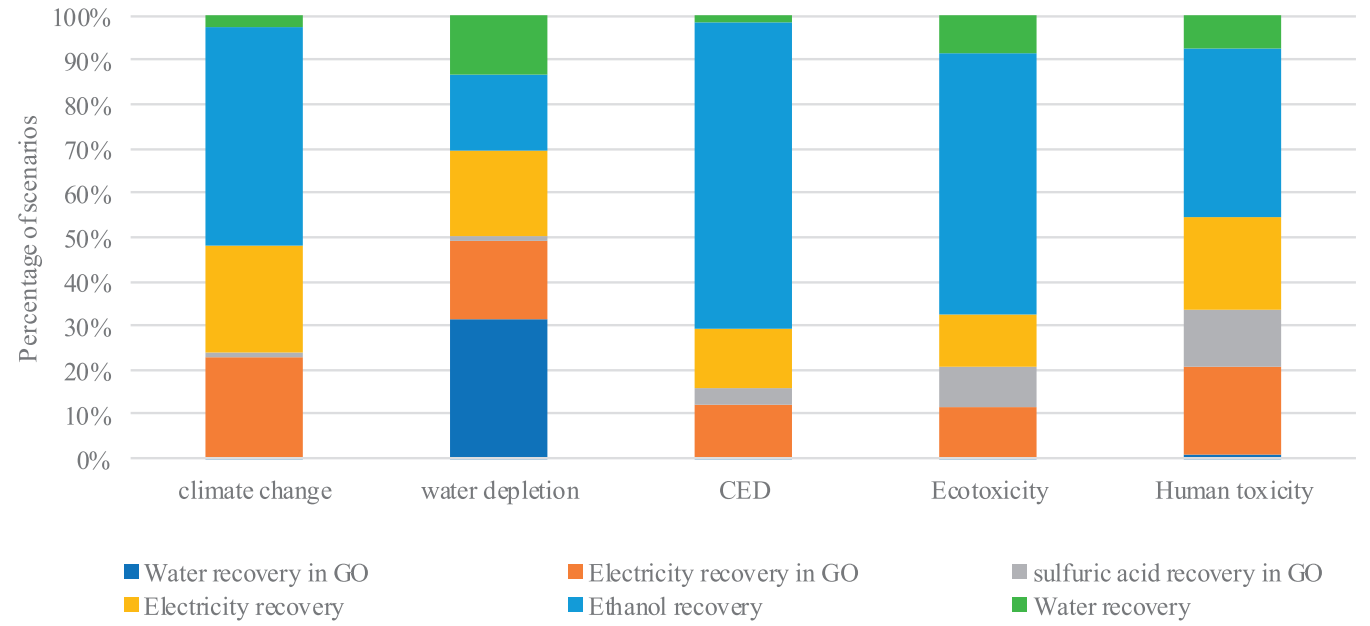

Fig. 4. The results of the sensitivity analysis for N-rGO synthesized by AM, showing the importance of the single scenarios for the reduction of each impact category in percentage.

depletion, and improving the efficient of sulfuric acid recovery in GO can efficiently decrease the impacts of ecotoxicity and human toxicity.

Electricity reduction in N-rGO synthesized is considered in sensitivity analysis. For HM, it can contribute to the largest impact reduction for five impactors in all sensitivity scenarios, which is from $48.5 \%$ to $67 \%$ for baseline scenario, this is because in HM a large amount electricity is needed for hydrothermal reaction. The largest impact reduction is the climate change, and then is human toxicity, water depletion, CED and ecotoxicity, respectively. So improving the efficient of electricity reduction is the main way for HM to reduce the environmental impacts, especially reducing the electricity use for hydrothermal reaction. For AM, the influence of electricity reduction is less than that in $\mathrm{HM}$, it is similar to the electricity reduction in GO. The reduction of impacts is from $6.3 \%$ (for ecotoxicity) to $13.5 \%$ (for climate change), which is also a good way for AM to reduce the environmental impacts.

Ethanol recovery is also an important parameter for two methods. For HM, ethanol recovery can decrease the impacts from $6.6 \%$ (for water depletion) to $32.2 \%$ (for CED), similarly the impact of ecotoxicity also has a big reduction (approximately 27.5\%). Thus, improving the efficient of ethanol recovery can efficiently decreased all environmental impacts. For AM, except water depletion, ethanol recovery is the largest factor for other four environmental impacts. The largest is CED (approximately $36.0 \%$ for baseline scenario), and then are ecotoxicity (31.1\%), climate change $(27.7 \%)$, human toxicity (21.8) and water depletion (11.9\%). Therefore, improving the efficient of ethanol recovery is the most efficient way to decrease the environmental impacts for N-rGO synthesized by AM.

From the sensitivity analysis results, the water recover is relative small factor for two methods to 
reduce the environmental impacts. Especially for $\mathrm{HM}$, the largest contribution is $4.9 \%$ reduction for water depletion, others are all less than 4\%. For AM, similarly the largest contribution is $8.9 \%$ reduction for water depletion, others are all less than 5\%. Thus, improving the water recovery can decrease the water depletion in two methods, and the contribution for other environmental impacts is negligible.

We also assess the dosage of urea in HM, the results show the different dosages of urea for all environmental impacts are negligible, the reductions in all environmental impacts are less than $1.2 \%$ for baseline scenario.

\section{Conclusion}

The results of the study show that all the environmental impacts in our study of HM is higher than that of $\mathrm{AM}$, so $\mathrm{AM}$ route is more available to scale-up production than HM route. For almost all environmental impacts in two routes, electricity, ethanol and water use are mainly contributor, the impact of the nitrogen precursors can be negligible. The sensitivity analysis results show that for GO production, improving the efficient of water recovery is good way to reduce the impact of water depletion, and the impacts of sulfuric acid recovery and electricity reduction are relatively small, especially in AM which can be negligible. For $\mathrm{AM}$, electricity reduction is the most efficient way to decrease the environmental impacts, and the next is ethanol recovery. For HM, ethanol recovery is the most efficient way to decrease environmental impacts, and the next is electricity reduction.

\section{Acknowledgements}

This study was supported by the China Scholarship Council.

\section{References}

1. TUGBA O-H, IDIL A.-A., SEBAHATTIN G., ILAHA G., SHIVA K., SERZAT S., DUYGU Y.O. Oxidative degradation of Bisphenol A by carbocatalytic activation of persulfate and peroxymonosulfate with reduced graphene oxide. Journal of Hazardous Materials, 360 (15), 141, 2018.

2. YADAV R., DIXIT C.K. Synthesis, characterization and prospective applications of nitrogen-doped graphene: A short review. Journal of Science: Advanced Materials and Devices, 2 (2), 141, 2017.

3. WANG H., MAIYALAGAN T., WANG X. Review on recent progress in nitrogen-doped graphene: synthesis, characterization, and its potential applications. ACS Catalysis, 2 (5), 781, 2012.

4. KONG X.-K., CHEN C.-L., CHEN Q.-W. Doped graphene for metal-free catalysis. Chemical Society Reviews, 43 (8), 2841, 2014
5. ZHENG Y., JIAO Y., GE L., JARONIEC M. Qiao SZJACIE: Two-step boron and nitrogen doping in graphene for enhanced synergistic catalysis. Angewandte Chemie, 52 (11), 3110, 2013.

6. DUAN X., SUN H., WANG S. Metal-free carbocatalysis in advanced oxidation reactions. Accounts of Chemical Research, 51 (3), 678, 2018.

7. DUAN X., AO Z., SUN H., INDRAWIRAWAN S., WANG Y., KANG J., LIANG F., ZHU Z.H., WANG S. Nitrogendoped graphene for generation and evolution of reactive radicals by metal-free catalysis. ACS Applied Materials \& Interfaces, 7 (7), 4169, 2015.

8. DUAN X., INDRAWIRAWAN S., SUN H., WANG S. Effects of nitrogen-, boron-, and phosphorus-doping or codoping on metal-free graphene catalysis. Catalysis Today, 249, 184, 2015.

9. KANG J., ZHOU L., DUAN X., SUN H., WANG S. Catalytic degradation of antibiotics by metal-free catalysis over nitrogen-doped graphene. Catalysis Today, 2018.

10. KANG J., DUAN X., ZHOU L., SUN H., TAD ${ }^{\circledR}$ M.O., WANG S. Carbocatalytic activation of persulfate for removal of antibiotics in water solutions. Chemical Engineering Journal, 288, 399, 2016.

11. LUO Z., LIM S., TIAN Z., SHANG J., LAI L., MACDONALD B., FU C., SHEN Z., YU T., LIN J Pyridinic $\mathrm{N}$ doped graphene: synthesis, electronic structure, and electrocatalytic property. Journal of Materials Chemistry, 21 (22), 8038, 2011.

12. ZHANG C., FU L., LIU N., LIU M., WANG Y., LIU Z Synthesis of nitrogen-doped graphene using embedded carbon and nitrogen sources. Advanced Materials, 23 (8), 1020, 2011.

13. DENG D., PAN X., YU L., CUI Y., JIANG Y., QI J., LI W.-X., FU Q., MA X., XUE Q Toward N-doped graphene via solvothermal synthesis. Chemistry of Materials, 23 (5), 1188, 2011.

14. PANCHAKARLA L., SUBRAHMANYAM K., SAHA S., GOVINDARAJ A., KRISHNAMURTHY H., WAGHMARE U., RAO C. Synthesis, structure, and properties of boron- and nitrogen-doped graphene. Advanced Materials, 21 (46), 4726, 2009.

15. GENG D., CHEN Y., CHEN Y., LI Y., LI R., SUN X., YE S., KNIGHTS S. High oxygen-reduction activity and durability of nitrogen-doped graphene. Energy and Environmental Science, 4 (3), 760, 2011.

16. JEONG H.M., LEE J.W., SHIN W.H., CHOI Y.J., SHIN H.J., KANG J.K., CHOI J.W. Nitrogen-doped graphene for high-performance ultracapacitors and the importance of nitrogen-doped sites at basal planes. Nano Letters, 11 (6), 2472, 2011.

17. LONG D., LI W., LING L., MIYAWAKI J., MOCHIDA I., YOON S.-H. Preparation of nitrogen-doped graphene sheets by a combined chemical and hydrothermal reduction of graphene oxide. Langmuir, 26 (20), 16096, 2010.

18. TISCHNER U., DEUTSCHLAND U. How to do EcoDesign?: a guide for environmentally and economically sound design. Verlag form, 2000.

19. SHEIKH M.M., ROKSANA M., KAREN H., MICHAEL C.-D. Life cycle assessment of emerging technologies: A review. Journal of Industrial Ecology, 24 (1), 52, 2020.

20. KARN B.J. The road to green nanotechnology. Journal of Industrial Ecology, 12 (3), 263, 2008.

21. COSSUTTA M., MCKECHNIE J., PICKERING S.J. A comparative LCA of different graphene production routes. Green Chemistry, 19 (24), 5874, 2017. 
22. ARVIDSSON R., KUSHNIR D., SANDEN BÊA, MOLANDER S. Prospective life cycle assessment of graphene production by ultrasonication and chemical reduction. Environmental Science \& Technology, 48 (8), 4529, 2014.

23. KAZEMI A., BAHRAMIFAR N., HEYDARI A., OLSEN S.I. Life cycle assessment of nanoadsorbents at early stage technological development. Journal of Cleaner Production, 174, 527, 2018.
24. HETHERINGTON A.C., BORRION A.L., GRIFFITHS O.G., MCMANUS M.C. Use of LCA as a development tool within early research: challenges and issues across different sectors. International Journal of Life Cycle Assessment, 19 (1), 130, 2014.

25. BRADDER P., LING S.K., WANG S., LIU S.J. Dye adsorption on layered graphite oxide. Journal of Chemical \& Engineering Data, 56 (1), 138, 2010. 